\title{
An Intelligent Edge Computing based Semantic Gateway for Healthcare Systems Interoperability and Collaboration
}

\author{
Tshiamo Sigwele, Yim Fun Hu, \\ Muhammad Ali and Jiachen Hou \\ University of Bradford \\ Bradford, United Kingdom \\ Email:[T.Sigwele1, Y.F.Hu, \\ M. Ali70, J.Hou1]@bradford.ac.uk
}

\author{
Misfa Susanto and Helmy Fitriawan \\ Department of Electrical Engineering \\ Faculty of Engineering \\ University of Lampung, Indonesia \\ Email: misfa@eng.unila.ac.id \\ Email: helmy.fitriawan@eng.unila.ac.id
}

\begin{abstract}
The use of Information and Communications Technology (ICTs) in healthcare has the potential of minimizing medical errors, reducing healthcare cost and improving collaboration between healthcare systems which can dramatically improve the healthcare service quality. However interoperability within different healthcare systems (clinics/hospitals/pharmacies) remains an issue of further research due to a lack of collaboration and exchange of healthcare information. To solve this problem, cross healthcare system collaboration is required. This paper proposes a conceptual semantic based healthcare collaboration framework based on Internet of Things (IoT) infrastructure that is able to offer a secure cross system information and knowledge exchange between different healthcare systems seamlessly that is readable by both machines and humans. In the proposed framework, an intelligent semantic gateway is introduced where a web application with restful Application Programming Interface (API) is used to expose the healthcare information of each system for collaboration. A case study that exposed the patient's data between two different healthcare systems was practically demonstrated where a pharmacist can access the patient's electronic prescription from the clinic.
\end{abstract}

Index Terms-Internet of Things, Healthcare Interoperability, Smart Gateway, Semantic Web, Ontology, Sensor Networks

\section{INTRODUCTION}

Recently, there has been a paradigm shift in the healthcare sector as many healthcare organisations have gradually migrated paper-based patient medical records to digital electronic ones by the implementation of Electronic Health Records (EHR) systems [1]. The recorded information can be observations, patient's personal details, patients medical history, clinical notes, laboratory tests, treatments, drugs administered, letters, x-rays, and bills [2]. The EHR systems can prevent duplicative tests by sharing patient information between medical facilities, which would inevitably result in cost-saving and also improved healthcare quality. With EHR, healthcare organisations can share patient's healthcare information with each other; therefore, they are able to make

This work was conducted within the framework of the BLESS U project, funded through a British Council Institutional Links grant under the BEIS-managed Newton Fund.

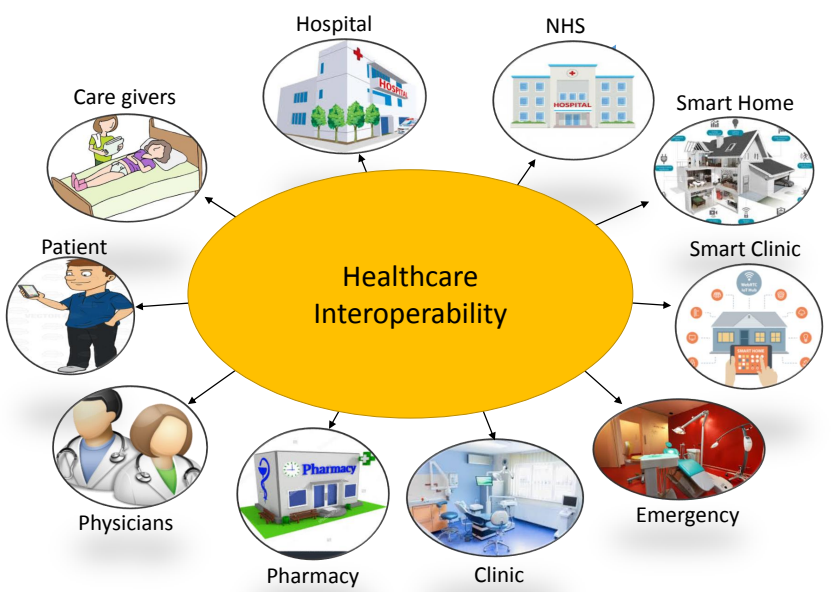

Fig. 1. An illustration of Healthcare interoperability.

better healthcare decisions. The information to be shared is stored in heterogeneous distributed healthcare systems, in different file formats which are mainly proprietary [2]. These information need to interact and be accessed by healthcare systems in a uniform and transparent way, anywhere and anytime. However, even with the introduction of EHR, healthcare systems are still isolated from each other with the lack of collaboration and interoperability. As shown in Fig. 1 , healthcare interoperability can be defined as the ability of two or more distinct healthcare systems like smart hospitals, clinics, smart homes, pharmacy etc. to share the information reliably and quickly from other each other in order to operate on them together without the occurrence of errors hence improving availability [3]. Healthcare interoperability has the following advantages [2]:

i) Easy access of patients records

ii) Reduction of medical errors hence less casualties.

iii) Healthcare cost reduction

iv) Reducing delays in medal healthcare systems

It has been estimated that in the next 10 years, the way 
healthcare is currently provided will be transformed from hospital centred, first to hospital home balanced in 2020, and then ultimately to home-centred in 2030 [4]. This essential transformation necessitates the fact that the convergence and overlap of the Internet of Things (IoT) architectures and technologies for smart spaces and healthcare domains should be more actively considered. The IoT in healthcare will also escalate the generation of large amounts of healthcare data and there is a need for collaboration by healthcare systems to share this healthcare big data.

This paper proposes a semantic based healthcare collaboration framework that is able to offer a cross system information exchange platform between different healthcare systems seamlessly that is readable by both machines and humans. The healthcare systems considered are heterogeneous and geographically distributed pharmacy, smart homes, smart hospitals and smart clinics that need to interact and collaborate with each other. In the proposed framework, each healthcare system incorporates an intelligent semantic smart gateway and a web application with restful Application Programming Interface (API) that is used to securely expose the information of each healthcare system for collaboration. To validate our claim, a case study is also presented to show the concept on interoperability between a pharmacy and a clinic using ontology.

The rest of the paper is organised as follows: Section II discusses the related work in interoperability within the healthcare sector and some common healthcare standards. Section III presents the proposed semantic-based healthcare Interoperability framework. The proposed healthcare system architecture, the functions of the semantic smart gateway and an ontology based case study are also presented in Section III. Finally, concluding remarks are presented in Section IV and Aknowledgement in Section V.

\section{RELATED WORK}

To solve the problems of independence existing among the healthcare systems, it is indispensable to implement interoperability in these systems. There have been several efforts in the health sector to address interoperability issues however, this remains an open issue for further research. The health data complexity, healthcare security and standardisation gives rise to healthcare systems integration difficulties and interoperability problems. A way to solve these problems is to use a common data model [3].

Healthcare standards provide the base for interoperability between different healthcare systems [5]. The healthcare sector has formulated appropriated standards for different purposes. The standards relate to how healthcare messages are relayed, the terminology used, the documents, conceptual frameworks, architectures and applications, both for syntactic interoperability, based on the communication structure, and for semantic interoperability, which defines the meaning of the communication. Some of the common healthcare standards are as follows [5]; messages related standards by Health Level Seven (HL7) International like HL7, HL7 V2 and V3 [6] which are considered the more adaptable standards to healthcare interoperability; terminologies (Systematised Nomenclature of Medicine Clinical Terminology SNOMED-CT [6]); clinical information and patients records (openEHR [7] and HL7 Clinical Document Architecture-CDA).

The authors in [8] proposed a fog-based IoT-Healthcare solution structure and explore the integration of cloud-fog services in interoperable healthcare solutions extended upon the traditional cloud-based structure. The scenarios were evaluated through simulations using the iFogSim simulator and the results were analysed in relation to distributed computing, reduction of latency, optimisation of data communication, and power consumption. However, the authors only integrated various IoT devices within a single healthcare system but did not provide interoperability between various healthcare systems. The authors in [9] proposed semantic interoperability model for big-data in IoT (SIMB-IoT) to deliver semantic interoperability among heterogeneous IoT devices in the healthcare domain. This model is used to recommend medicine along with their side effects for different symptoms collected from heterogeneous IoT sensors. However only one healthcare system is considered and interoperability is only among heterogeneous IoT devices.

Jini Health Interoperability Framework (HIF-J) proposed in [10] uses Jini technology which is based on SOA. The main purpose of HIF-J is to exchange semantically interoperable messages. It provides translation services that behaves as a mediator between standards. These translation services convert message instances of HL7, HL7 V2 and V3 and also openEHR message instances. It is based on extensible Stylesheet Language Transformations (XSLT) between message instances of different standards. Since standards are growing with new domains, so managing XSLT becomes very difficult. In [11], the authors focus on semantic process interoperability with the help of interaction ontology in HL7 V3. Interaction ontology is responsible for handling the heterogeneities between processes of different healthcare systems compliant to HL7 V3 standard. This work is only related to semantic process interoperability using standard HL7 V3 and semantic data interoperability is not discussed. The authors in [15] used ontology matching tools to resolve the data level heterogeneities between different healthcare standards and achieve message schema level conversion; however, the author only concentrated on only HL7 healthcare standards. The authors in [12] proposed a Smart e-Health Gateway capable of enhancing IoT architectures used for healthcare applications integrating sensor healthcare data from a hospital to a cloud healthcare centre, however, the framework did not provide interoperability between distinct healthcare systems.

From the current literature, efforts to provide interoperability in healthcare systems only concentrate on various sections of a single healthcare system but they do not provide means of integration with other different health system bodies. Also, interoperability in the current research mostly concentrate on information exchange but 
omit the sharing and exchange of the semantics knowledge of information and its relationship.

\section{Proposed Semantic based HealthCare INTEROPERABILITY FRAMEWORK}

In this section, the proposed architecture will be presented, then collaboration framework will be explained and finally a case study presented and practically addressed to show how ontology can be used to share information between independent healthcare systems.

\section{A. Proposed Healthcare System Architecture}

The proposed architecture of the healthcare system which can be used for interoperability is shown in Fig. 2 (a). The patient health related information to be exchanged is to be collected from various sources like other healthcare systems, from the web like DBPedia, Wikipedia or recorded by smart devices or body-worn sensors with which the patient is equipped for personal monitoring of multiple parameters. The system architecture comprises of smart devices, fog layer and cloud layer as shown in Fig. 2 (b).

1) Smart Devices: Smart devices include smart cameras, body sensors, wearable devices, and other various sensors. The majority of these devices, for example wearable medical sensors, are not capable of storing data they generate. A straightforward design approach is to transfer their data to a remote cloud for processing. Given the large number of connected devices, the latency of the connection with the cloud could be significant. Moreover, these devices are power and bandwidth constrained, that make them unfit directly to the cloud architecture. Edge Computing is an essential paradigm shift towards a hierarchical system architecture and a more responsive design and therefore adopted in this paper. The concept behind the semantic smart gateway is to provide different services at the edge of the network between the smart objects and the cloud. The data from the smart device is then transmitted to the gateway via wired or wireless communication protocols.

2) Fog Layer: The fog layer connects smart devices to the internet and is situated at the edge of the network. It consists of the semantic smart gateway, which forms the core part of the proposed framework where all the business logic takes place which will be explained further in Section III. The gateway supports various wireless protocols and inter-device communication. The health sensors and the context (temperature, light sensor, flood sensor etc.) sensors are connected to the gateway using either wireless network or wired connections of different standards (e.g., 6LoWPAN, LoRa, Sigfox, NB-IoT, WiFi etc.). The smartness of the gateway comes in the form of easy integration of these heterogeneous networking technologies, protocols and standards with multiple interfaces thereby enabling them to exchange information and work seamlessly. Apart from supporting heterogeneous protocols and performing protocol conversion, the semantic gateway also performs other functions including data capture, representation and modelling, data processing and knowledge generation, data filtering, data analysis etc. It also acts as an information dissemination point to other healthcare systems via the REST API.

3) Cloud Layer: This is the internet where all other healthcare system parties can access information and data. It consists of the remote cloud data centres, which host the knowledge base of the proposed framework. These data centres also store the generated semantic information in Resource Description Framework/ eXtensible Markup Language (RFD/XML) files, which are written in Web Ontology Language (OWL). OWL files can be viewed as databases of the future. They not only store the data of a system but also the metadata (data about data) called semantics which include relationships between concets. A large number of OWL files with individual instances of classes forms the knowledge base which can act as a database of any application. The cloud layer also comprises of online tools and repositories like wikipedia, Xively, DBpedia and ThinkSpeak and all other connected healthcare systems (emergency, pharmacy, National Health Service (NHS)) which can collaborate and request information seamlessly from other systems via the proposed framework. A web client is used as a graphical user interface for final visualisation and feedback. The web client can be accessed only by authorised users of other healthcare system.

\section{B. Proposed Healthcare Systems Collaboration Framework}

The proposed healthcare system collaboration framework is shown in Fig. 3. The purpose of the collaboration framework in the context of the healthcare is to: (1) capture and represent data from various sources (sensors or other healthcare systems via the web), (2) generate knowledge, and (3) share and exchange information and knowledge with other external healthcare systems. Data capture and representation is the first component of the framework. This is where acquisition of raw data and its modelling and representation takes place. The data will usually be modelled according to a proprietary schema. The next step is to apply semantic contexts and business rules on the data to convert it into useful information and actionable knowledge. Finally, the processed knowledge is ready to be exposed and collaborated upon with external healthcare agents through an API.

1) Data capture, representation and modelling: Healthcare data is primarily captured from local sensor networks, but it can also be fetched from repositories that expose their data through an API (e.g. Xively). The asset model handles the modelling and representation of the sensing devices and data in a platform-specific manner and is stored according to a proprietary schema. Data may be stored in any corresponding storage medium like Relational Database Management System (RDBMS), XML files, Comma-Separated Values (CSV) files, excel files, etc. The asset model makes data available to the other components of the collaboration framework for further processing and enrichment. The access management component deals with the authentication and authorisation of actors who want to access the data stored in the asset model. These actors can be 


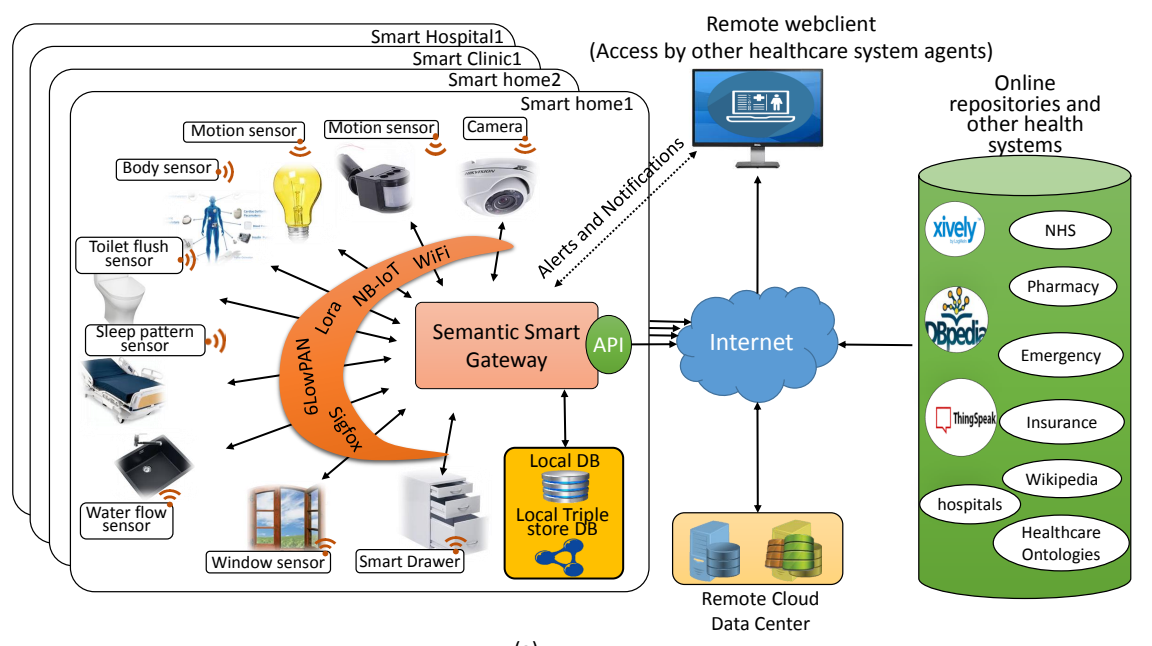

(a)

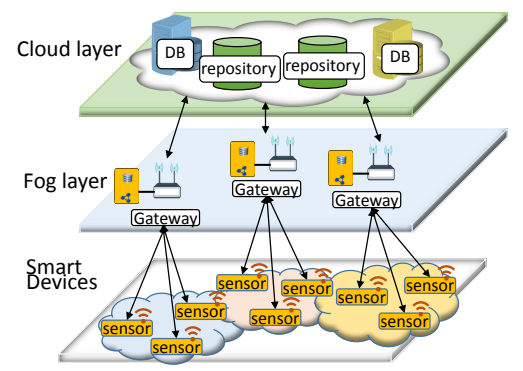

(b)

Fig. 2. (a) The proposed IoT based healthcare interoperability architecture (b) Layers in the proposed architecture.

both internal (administrators) and external (other healthcare systems).

2) Data processing and knowledge generation: Once the data has been captured from the local sensor network or imported from other repositories, this data needs to be contextualised so that it can represent some meaningful information. The process of turning raw data into useful information can take place through a variety of methods. As an example, business rules can be applied to pieces of data to generate meaningful information. For example, a numeric data value taken from a sensor can have semantic contexts applied to it so that it turns into useful information, like a blood pressure level or water flow level. This whole field of capturing data and then generating, annotating and making available high-level information is known as Knowledge Management (KM). For each healthcare system, various data sources from the asset model are mapped into a local ontology such that each healthcare system has a local ontology. The local ontologies are then mapped into one global ontology which all the collaborating healthcare systems are built from. The ontologies are stored in .owl file formats in the internet and can be accessed and understood by machines and humans.

3) Semantic Gateway Add-ons: The gateway is also incorporated with some additional add-ons to be performed at the network edge as follows:

i) Local Storage: Data storage on gateways brings system reliability especially when the network is unavailable. The gateway proposed enables smooth data recover since it stores data locally in compressed or encrypted form. Data in the repository can be exported to medical standard formats such as HL7,HL7 V2 or HL7 V3 if required. The gateway is responsible for data analysis, compression, filtering, and encryption and all these functions requires a local temporary storage rather than sending parameters to a cloud and waiting for the responses. Consequently, the healthcare system reacts to the emergency situation much faster with real-time responses. Moreover, it is possible to save the sensory data and processing results in a local storage at the fog layer and synchronise them with the Cloud later.

ii) Security: An unsecured systems can have serious vulnerabilities and security should be considered as one of the most essential requirements in health applications. As the gateway can also act as an embedded web server during network unavailability, it can communicate over secure Hypertext Transfer Protocol Secure (HTTPS) and authenticate sensor nodes to maintain the confidentiality, integrity, and authenticity of the system.

iii) Data Analysis: Real-time monitoring and analysis of the healthcare system can be implemented using a real-time server powered by Node.js as in [13] which has few additional key functions that enable real-time monitoring and analysis of the network as well as real-time capture and publication of assets. The sensitivity of the system is improved by applying local data analysis at the edge. It can assist the system to detect and predict emergency situations. For instance, in case of fall detection for in a smart home, the fog layer can locally offer fall-detection related processing

iv) Data Compression: In the context of data communication, data compression is used for minimising communication latency and energy consumed during transaction.

v) Standardisation: Depending on where data is sent, data can be formated into any of the healthcare standards such as HL7, HL7 V2, HL7 V3 when necessary by the smart gateway, in the standardisation module. Sensor nodes can be free from processing overhead that results in formatting the data into standards. In addition, the overhead on the communication channel due to the standards related information that could be sent with the data is removed

4) Collaboration: After the high level information has been generated and ontologies has been built, the information 


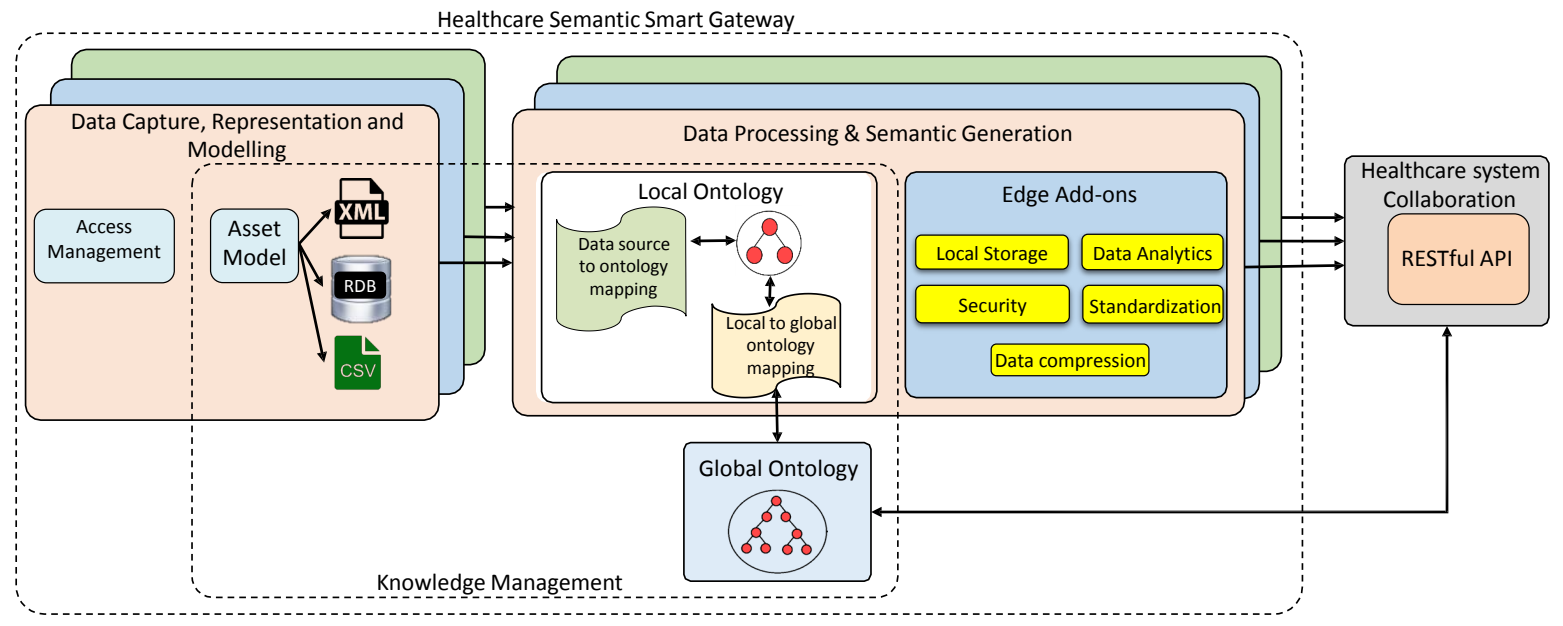

Fig. 3. Proposed healthcare systems collaboration framework.

and the knowledge of the healthcare system can then be exposed to external healthcare systems. An API is developed to enable collaboration since it can expose information that is represented in either a proprietary or an interoperable fashion. The front-end web application can be hosted on an Apache webserver and exposes the underlying functionalities through a RESTful API (Application Programming Interface). A webapp provides a Graphical User Interface (GUI) and exposes the semantic engine for applications like semantic querying of assets.

\section{Preliminary Ontology Case Study}

A case study is considered where two independent healthcare systems are built using OWL to share information. OWL is a semantic web language designed to represent rich and complex knowledge about things, groups of things, and relations between things. In the case study, there are two distinct healthcare systems not interacting with each other; 1) the clinic and 2) the pharmacy. A patient needs to first register with his/her local clinic and provides patient-related information, for example the patient's firstname, surname, patientID, etc.. After registration, a patient can book an appointment with the doctor in the local clinic through the web, telephone or in person. The doctor, during the appointment, diagnoses the sickness and makes a prescription for the patient. The patient now has a prescription which can treat his/her sickness. The clinic does not provide the medication list in the prescription as such, the prescription is printed and the patient is referred to the pharmacist to collect the medication. The patient can lose the paper prescription which may cause delays in treatment and hence serious health risks. At the pharmacy, the patient presents the prescription paper to the pharmacist and medication is then given without a more valid proof of the source of the prescription. Sometimes handwritten prescriptions gives the pharmacist's hard times to understand. Sometimes the medication is out of order and the patient has to wait for some few days to collect it which can worsen the medical condition.

It will be wise for the pharmacy to access the electronic prescription from the GP/doctor in the clinic automatically and prepare the list of medications in advance to eliminate the above problems of paper prescription. This means the clinic will expose only its prescription and the unique identifier of the patient like full name or patientID to the pharmacy in a secure manner e.g. using login and password to access the prescription notification after the patient has been given a prescription.

1) Ontology creation for the case study: The graphical view of the proposed ontologies to be created is shown in Fig. 4. Using OWL, interoperability of the clinic-pharmacy case study can be achieved smoothly by exposing the electronic prescription from the clinical doctor to the pharmacist. To address this case study, the clinic and the pharmacy healthcare systems will have their own ontologies written in OWL based on a shared ontology with global vocabulary. The ontologies are created with Protege, a free open-source ontology editor and framework for building ontologies. The clinic ontology created is called clinic.owl (Fig. 5) and the pharmacy ontology is called pharmacy.owl (Fig. 6). These are RDF/XML files that can be uploaded to the internet. To be precise, the clinic ontology Uniform Resource Identifier (URI) is http://www.semanticweb.org/tsigwel1/ontology $2 /$ clinic and the pharmacy ontology URI is http://www.semanticweb.org/tsigwel1/ontologies/pharmacy.

An online ontology viewer called webvowl can be used to graphically view the ontology at http://www.visualdataweb.de/webvowl. The global ontology is common to both systems and consist of a base class called Thing, where all other classes are derived from. The pharmacy ontology consists of 4 classes (thing, person,pharmacist, patient and prescription). The clinic ontology consists 8 classes (thing, person, patient, doctor, sickness, prescription, receptionist and appointment. 


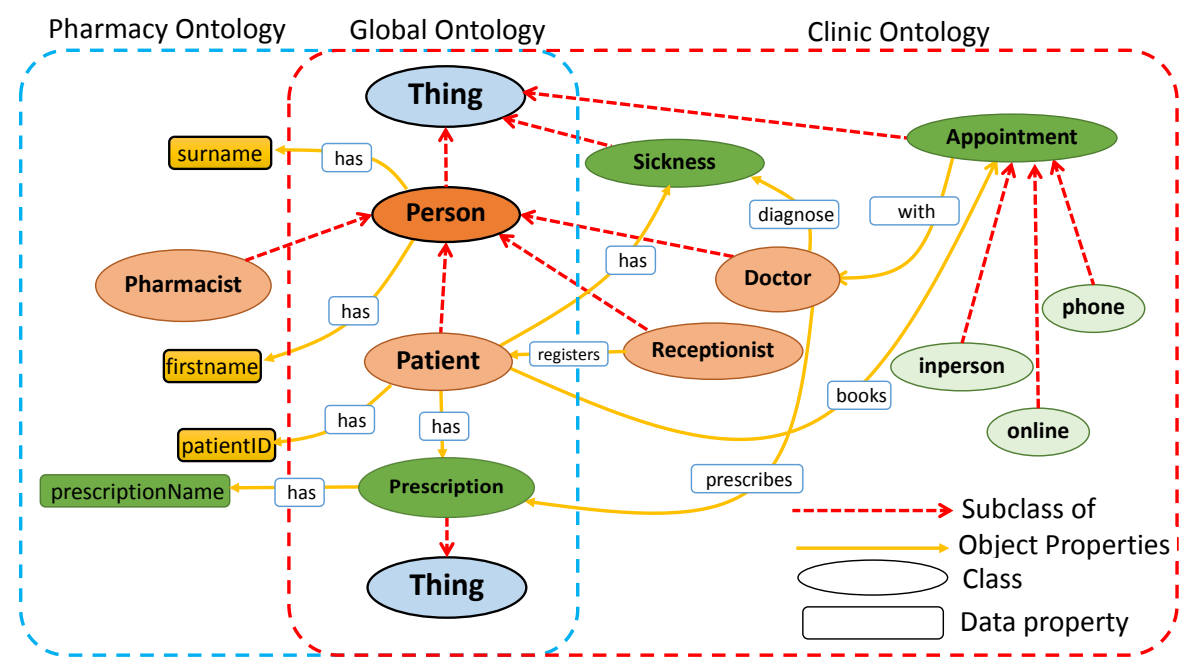

Fig. 4. Ontologies of the two systems with share global ontology enabling collaboration

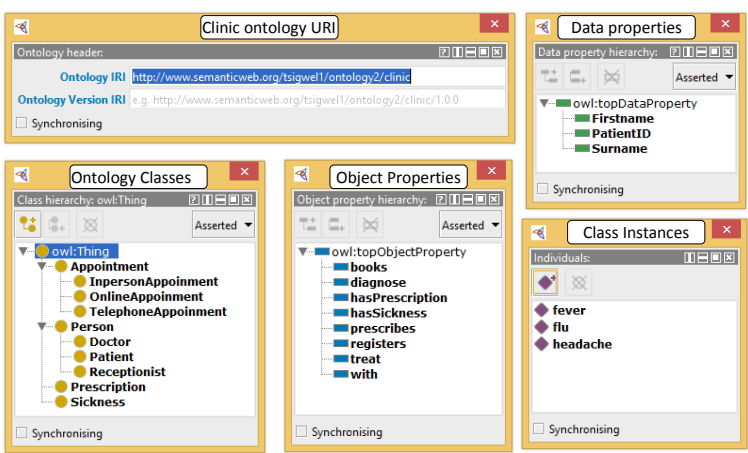

Fig. 5. Creation of clinic.owl ontology with Protege using OWL

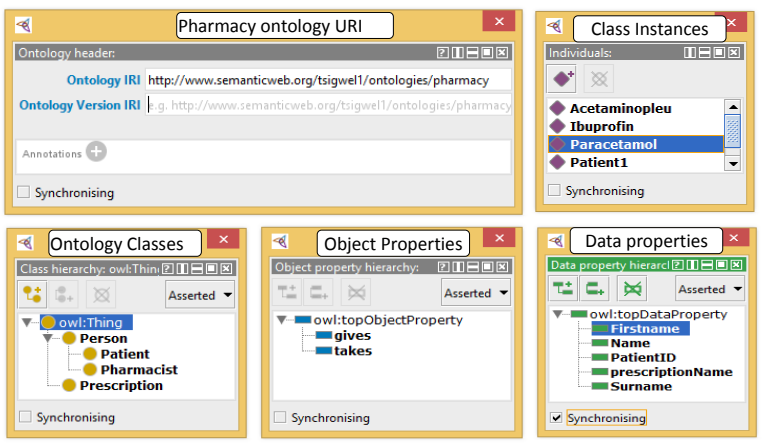

Fig. 6. Creation of pharmacy.owl ontology with Protege using OWL.

The classes are associated with each other by object properties e.g., Patient 'books' Appoinment where books is an object property. It can be clearly seen in Fig. 4 that there are some common concepts/casses between the two systems like (patient, prescription). With ontology, this concepts are standardised such that they are of the same format to both systems which makes the instances of the shared classes like 'patient1' and 'prescriptionForPatient1' to be accessed, opened and viewed by the other party with ease. After the ontologies are created, the clinic exposes the Patient details and the 'prescriptionName' through the web. Fig. 7(a) and Fig. 7(b) show part of the pharmacy and clinic ontologies in RDF/XML format.

2) Clinical data dissemination to the Pharmacy: Fig. 8 shows a pharmacist accessing the electronic version of the prescription from the clinic. The clinic exposes its patient and prescription data using a SPARQL Endpoint where the pharmacist with access rights can querry the clinic.owl which stores the data of the clinic. A SPARQL endpoint is where SPARQL query language is used to search data stored in the clinic.owl ontology file created at the pharmacy side for accessing the prescription. The SPARQL endpoints are simpler ways of querying a list of OWL files normally called knowledge base. The machines in the pharmacy area (reception computer and other pharmacy machines) can understand the prescription requested from the clinic and can validate the source of the prescription as the clinic is trusted and the future machines in the pharmacy can even dispense the prescrition to the patient automaticaly reducing delays and errors.

\section{CONCLusion}

This paper presented a conceptual semantic based healthcare collaboration framework that offers information exchange between different IoT healthcare systems seamlessly that is readable by both machines and humans. The healthcare systems considered are heterogeneous and geographically distributed smart homes, smart hospitals and smart clinics that need to interact and collaborate with each other. In the proposed framework, each healthcare system incorporated an intelligent edge semantic gateway consisting of a web application with a restful API that is used to securely expose the healthcare information of each healthcare system for collaboration. A case study that exposed the patient's data 


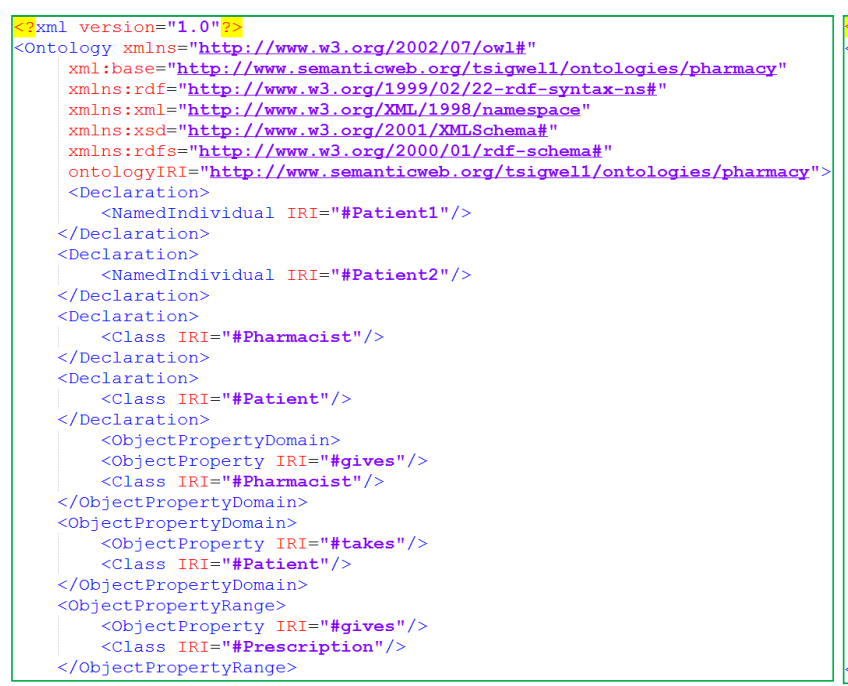

(a)

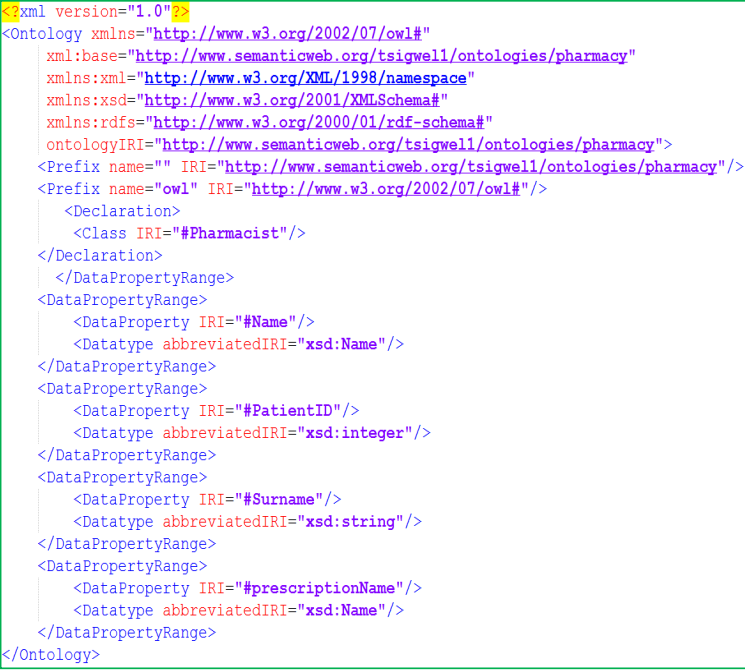

b)

Fig. 7. (a) Pharmacy ontology in RDF/XML format and (b) Clinic ontology in RDF/XML format.

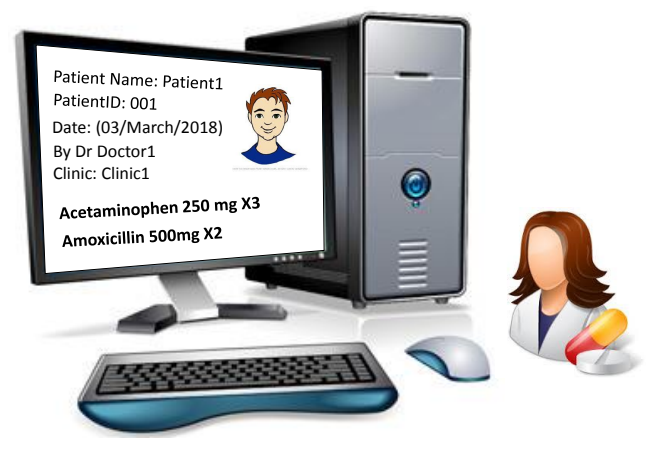

Fig. 8. Patient prescrition viewed by the pharmacist.

from other healthcare systems was practically demonstrated where a pharmacist can access clinical data stored in the knowledge base (OWL files). This preliminary work will be extended to a prototype by simulation where semantic web will be used and tool like Protege, Jena, iFogSim will be extensively used to build distinct multiple healthcare systems, build their local ontologies, then integrate the two systems by developing an RESTful API web app to expose the information and knowledge between the two systems.

\section{ACKNOWLEDGEMENT}

This work was conducted within the framework of the BLESS U (Bandar Lampung Enhanced Smart-health Services with Smart Ubiquity) project, funded through a British Council Institutional Links grant under the BEIS-managed Newton Fund.

\section{REFERENCES}

[1] T. Benson, Principles of health interoperability HL7 and SNOMED. Springer London:, 2010.
[2] O. Iroju, A. Soriyan, I. Gambo, and J. Olaleke, "Interoperability in healthcare: benefits, challenges and resolutions," International Journal of Innovation and Applied Studies, vol. 3, no. 1, pp. 262-270, 2013.

[3] L. Cardoso, F. Marins, F. Portela, M. Santos, A. Abelha, and J. Machado, "The next generation of interoperability agents in healthcare," International journal of environmental research and public health, vol. 11, no. 5, pp. 5349-5371, 2014.

[4] C. E. Koop, R. Mosher, L. Kun, J. Geiling, E. Grigg, S. Long, C. Macedonia, R. C. Merrell, R. Satava, and J. M. Rosen, "Future delivery of health care: Cybercare," IEEE Engineering in Medicine and Biology Magazine, vol. 27, no. 6, 2008.

[5] L. Cardoso, F. Marins, F. Portela, A. Abelha, and J. Machado, "Healthcare interoperability through intelligent agent technology," Procedia Technology, vol. 16, pp. 1334-1341, 2014.

[6] HL7-International, Introduction to HL7 Standards, 2018 (accessed March 19, 2018). [Online]. Available: http://www.hl7.org/implement/ standards/

[7] openEHR.org, An open domain-driven platform for developing flexible e-health systems, 2018 (accessed March 19, 2018). [Online]. Available: https://www.openehr.org/what_is_openehr

[8] R. Mahmud, F. L. Koch, and R. Buyya, "Cloud-fog interoperability in iot-enabled healthcare solutions," 2018.

[9] F. Ullah, M. A. Habib, M. Farhan, S. Khalid, M. Y. Durrani, and S. Jabbar, "Semantic interoperability for big-data in heterogeneous iot infrastructure for healthcare," Sustainable Cities and Society, vol. 34, pp. 90-96, 2017.

[10] A. J. Ducrou, "Complete interoperability in healthcare: technical, semantic and process interoperability through ontology mapping and distributed enterprise integration techniques," 2009.

[11] W. A. Khan, M. Hussain, M. Afzal, K. Latif, H. F. Ahmad, and A. Khattak, "Towards semantic process interoperability," in 10th International HL7 Interoperability Conference (IHIC), 2009, pp. 88-95.

[12] A.-M. Rahmani, N. K. Thanigaivelan, T. N. Gia, J. Granados, B. Negash, P. Liljeberg, and H. Tenhunen, "Smart e-health gateway: Bringing intelligence to internet-of-things based ubiquitous healthcare systems," in Consumer Communications and Networking Conference (CCNC), 2015 12th Annual IEEE. IEEE, 2015, pp. 826-834.

[13] M. Amir, Y. Hu, and P. Pillai, "A generic and extensible asset model for a semantic collaboration framework," International Journal of Advanced Computer Technology, vol. 3, no. 1, pp. 88-96, 2014. 